\title{
Acute interstitial nephritis, a potential predictor of response to immune checkpoint inhibitors in renal cell carcinoma
}

\author{
Viral Patel, ${ }^{1,2}$ Roy Elias (D) , , Joseph Formella (D) , ${ }^{1}$ William Schwartzman, ${ }^{1}$ \\ Alana Christie, ${ }^{2}$ Qi Cai, ${ }^{3}$ Venkat Malladi (1) , 2,4 Payal Kapur, ${ }^{3}$ Miguel Vazquez, ${ }^{1,5}$ \\ Renee McKay, ${ }^{1}$ Ivan Pedrosa, ${ }^{2,6}$ Raquibul Hannan (1) , ${ }^{1,7}$ Hans Hammers, ${ }^{1,2}$ \\ James Brugarolas (i) 1,2
}

To cite: Patel V, Elias R,

Formella J, et al. Acute interstitial nephritis, a potential predictor of response to immune checkpoint inhibitors in renal cell carcinoma. Journal for ImmunoTherapy of Cancer 2020;8:e001198. doi:10.1136/ jitc-2020-001198

VP and RE contributed equally.

Accepted 10 September 2020

Check for updates

(c) Author(s) (or their employer(s)) 2020. Re-use permitted under CC BY-NC. No commercial re-use. See rights and permissions. Published by BMJ.

For numbered affiliations see end of article.

\section{Correspondence to} Dr James Brugarolas; James.Brugarolas@ UTSouthwestern.edu

\section{ABSTRACT}

Immune checkpoint inhibitors (ICls) such as nivolumab and ipilimumab have improved outcomes in metastatic renal cell carcinoma (mRCC) patients, but they are also associated with immune-related adverse events (irAEs). As observed in melanoma, we hypothesized that patients experiencing an autoimmune reaction directed against the tissue of origin may be more likely to benefit from ICl. Specifically, we asked whether patients with immune-related acute interstitial nephritis (irAIN) exhibited improved outcomes. Using Kidney Cancer Explorer (KCE), a data portal and i2b2-based central database for clinical, pathological and experimental genetic data, we systematically identified all patients with $\mathrm{mRCC}$ at UT Southwestern Medical Center (UTSW) from 2014-2018 who received at least one dose of ICl. More recent cases were identified through a provider query. We extracted creatinine $(\mathrm{Cr})$ values at baseline and over the entirety of each patient ICl treatment course using KCE. Patients with $\geq 1.5$-fold $\mathrm{Cr}$ increase over baseline were investigated. The likelihood of irAIN was determined based on the workup (biopsy, if available), or by clinical criteria (timing of kidney injury, exclusion of other etiologies, treatment with immunosuppressants and response). We identified 177 mRCC patients who received at least one dose of $\mathrm{ICl}, 36$ of whom had $\geq 1.5$-fold increase in $\mathrm{Cr}$ over baseline while on treatment. Of those, two had biopsy-proven irAIN and one was clinically diagnosed, resulting in an incidence of $1.7 \%$. One additional biopsy-proven case past 2018 was identified through a provider query, for a total of four patients. Two received combination nivolumab and ipilimumab in the first line, whereas the remaining received nivolumab after first line therapy. irAIN onset ranged from 1.5 to 12 months. All four patients stopped ICI with recovery of renal function, at least partially, three after receiving systemic steroids. Notably, all four patients had a deep response. In conclusion, irAIN is a rare event, but it may portend a higher likelihood of response. One possible explanation is antigenic overlap between normal renal tubular cells and tumor cells.

\section{INTRODUCTION}

Renal cell carcinoma (RCG) is the ninth leading cause of cancer in the USA, with an estimated 73750 new cases and 14830 deaths in $2020 .^{1}$ The overall 5-year survival for all stages of RCC is $74.8 \%$; however, this number drops to $12 \%$ when the cancer has metastasized. ${ }^{1}$ Fortunately, outcomes for metastatic renal cell carcinoma (mRCC) are improving with the adoption of new therapies including immune checkpoint inhibitor (ICIs) and ICI/tyrosine kinase inhibitor combinations. ${ }^{2-5}$ ICIs used in the treatment of RCC target the PD1/PD-L1 and CTLA-4 immune checkpoints, and they are associated with immune-related adverse events (irAEs). ${ }^{2}{ }^{6}$ The incidence of irAEs can vary from $35 \%$ to $73 \%$ depending on the type and dose of ICI prescribed. ${ }^{6}$ The most commonly affected sites are the gastrointestinal tract, skin, liver, and endocrine organs; however, nearly every organ can be affected. ${ }^{6}$ Interestingly, irAEs following ICI therapy have been associated with improved outcomes in several observational studies, although the data are mixed and prospective studies are lacking. ${ }^{7}$ The mechanisms underlying irAEs are not yet entirely understood; however, studies suggest that disinhibition of autoregulatory T-cells and recognition of shared antigens may play a role. ${ }^{6-8}$ Consistent with the latter, several observational studies $^{9}{ }^{10}$ and a prospective clinical trial ${ }^{11}$ have linked the development of vitiligo with particularly good outcomes in patients with ICI-treated melanoma. Given the origin of RCG from renal epithelium, we hypothesized that immune-related acute interstitial nephritis (irAIN) may predict treatment efficacy in ICI-treated mRCC.

Here, we present the clinical outcomes of four cases of ICI-treated mRCC that developed irAIN at UT Southwestern Medical Center (UTSW). 


\section{METHODS}

We used Kidney Cancer Explorer (KCE), an Institutional Review Board-approved, i2b2-based queryable database that integrates clinicopathologic data automatically extracted from the electronic medical record (EMR) sponsored by the UTSW Kidney Cancer Program and the Lyda Hill Department of Bioinformatics Core Facility (manuscript in preparation). We collected comprehensive clinical and pathology data from patients with mRCC at UTSW who received at least one dose of ICI from January 1, 2014 until December 31, 2018. Creatinine (Cr) values prior to treatment initiation (baseline) and on treatment were automatically extracted, and patients with one or more $\mathrm{Cr}$ values demonstrating a $\geq 1$.5-fold increase over baseline during ICI therapy were investigated. More recent cases, diagnosed after the most recent KCE update (January 2019-February 2020), were identified by querying genitourinary oncology faculty at our institution. The diagnosis of irAIN was determined by renal biopsy, when available, or clinically if a biopsy was not available. Clinical inclusion criteria were defined as: (i) Cr rise after administration of ICI; (ii) exclusion of other causes of acute kidney injury (AKI), including prerenal, postrenal, or other intrinsic causes (including ischemia, sepsis, vasculitis, and nephrotoxin-induced AKI); and (iii) improvement following systemic steroid administration. Medical records for index patients were reviewed by at least two independent physicians (VP, RE) and a nephrologist (MV). Baseline characteristics were reported at the time of ICI initiation. Renal biopsies were reviewed by a qualified genitourinary pathologist (PK) and a nephrologist (MV). Response was assessed retrospectively using RECIST V.1.1 criterion by a qualified radiologist (IP).

\section{RESULTS}

A total of 177 patients with mRCC treated with at least one dose of ICI were identified from 2014 through 2018. Of these, $36(20.3 \%)$ patients had at least one $\mathrm{Cr}$ value that was $\geq 1.5$-fold over baseline while receiving ICI therapy. A total of 33 (18.6\%) patients were found to have non-irAIN etiologies of AKI including: 24 patients with prerenal injury resulting from poor fluid intake and/ or gastrointestinal losses; 7 patients who developed AKI in the context of a systemic illness (i.e., sepsis or acute heart failure); and 2 patients with postobstructive AKI (figure 1). irAIN was suspected in $3(1.7 \%)$ cases identified through the KCE search. One additional case diagnosed in 2019 was identified through a provider query. Three patients had renal biopsies confirming the irAIN diagnosis. The clinical characteristics of the four patients included in the study are summarized in table 1 .

\section{CASE PRESENTATIONS}

Case 1

The patient is a 71-year-old man with a medical history of hypertension (HTN), who initially presented in 2013 with a locally advanced tumor and a level II tumor thrombus. Radical nephrectomy and thrombectomy revealed clear cell (cc)RCC, nuclear grade (NG) 4 with $10 \%$ sarcomatoid features. Three months following resection, the patient was diagnosed with an isolated biopsy-proven iliac bone metastasis which was treated with stereotactic body radiotherapy (SBRT). Over a period of 2years, he developed four brain lesions amenable to focal therapy with resection of the largest $(1.8 \mathrm{~cm})$ metastasis and stereotactic radiosurgery (SRS) to the remaining subcentimeter lesions. Despite the

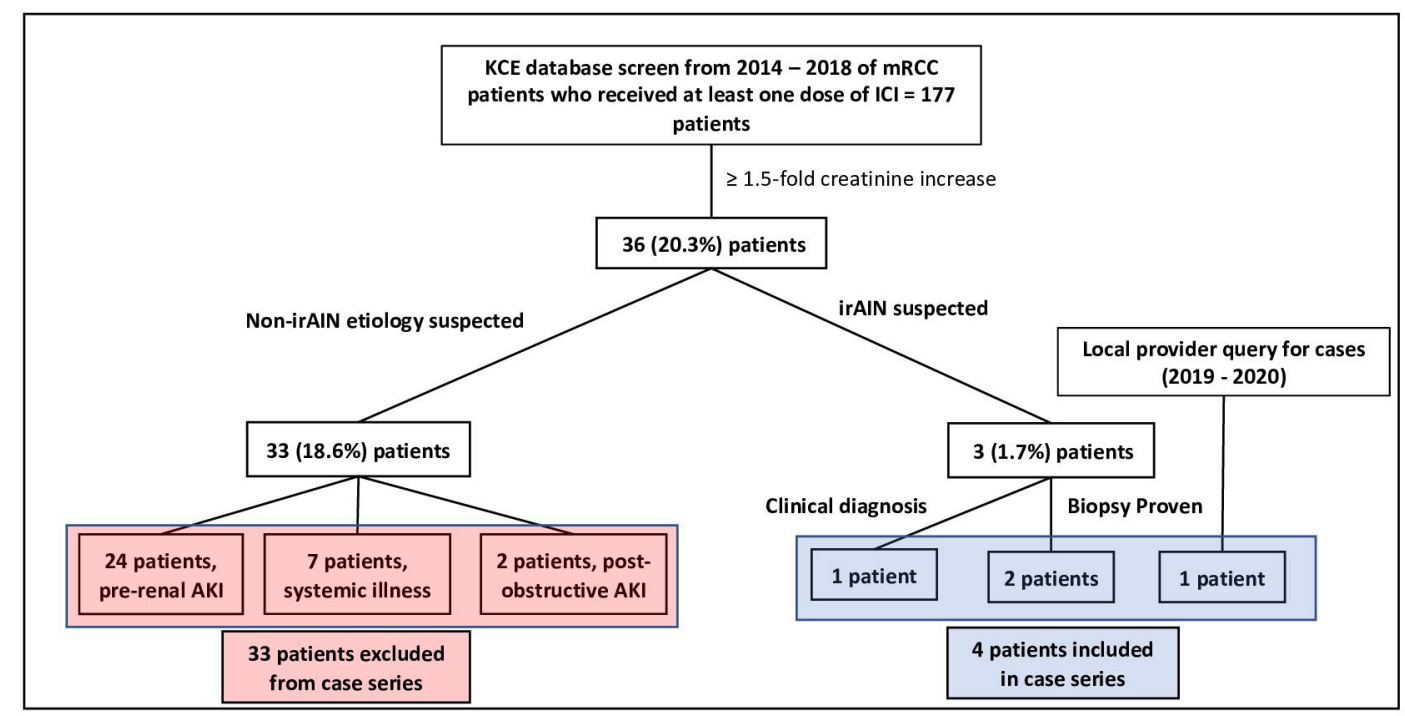

Figure 1 Study design and cohort identification. Patients with metastatic RCC treated with ICI were identified through a database that automatically extracts information from the medical record for cases treated prior to 2019 and a query of treating oncologists at UTSW for later cases. Medical records were reviewed, and patients with suspected non-irAIN mediated AKI were excluded. Four patients were ultimately identified with irAIN and included in this series. AKI, acute kidney injury; ICl, immune checkpoint inhibitor; irAIN, immune-related acute interstitial nephritis; KCE, Kidney Cancer Explorer; mRCC, metastatic renal cell carcinoma; UTSW, UT Southwestern Medical Center. 
brain metastases, the patient remained without extracranial disease until 2 years after his initial diagnosis. At this time, he developed several liver lesions, lung nodules, and a large paraesophageal lymph node metastasis, which was biopsied, confirming mRCC. The patient was started on pazopanib, but it was discontinued after 2 months due to hepatotoxicity with an increase in both transaminases and bilirubin. He was then started on nivolumab $3 \mathrm{mg} / \mathrm{kg}$ every 2 weeks. Over a period of 7 months, there was shrinkage of the paraesophageal lymph node metastasis as well as the pulmonary nodules (figure 2; compare A and B). At this time, the patient developed grade II pneumonitis which was treated with steroids and cessation of nivolumab. The pneumonitis resolved, and the patient transitioned to active surveillance. Unfortunately, 3 months later, the patient demonstrated progressive disease with enlargement of lung nodules prompting reinitiation of nivolumab (240 mg every 2 weeks). Two months later, the patient was found to have a Cr increase to $2.25 \mathrm{mg} /$ $\mathrm{dL}$ from $1.3 \mathrm{mg} / \mathrm{dL}$ (estimated glomerular filtration rate (eGFR) decrease to 35 from $55 \mathrm{~mL} / \mathrm{min} / 1.73 \mathrm{~m}^{2}$ ). A 24 hour urine collection revealed nephrotic range proteinuria, with $5017 \mathrm{mg}$ of protein, whereas earlier urinalyses had shown no evidence of proteinuria. Renal biopsy demonstrated interstitial nephritis, consistent with irAIN (figure 3). Treatment with nivolumab was stopped, and steroids were deferred per patient's preference. The patient's Cr gradually decreased to $1.7 \mathrm{mg} / \mathrm{dL}\left(\right.$ eGFR $\left.52 \mathrm{~mL} / \mathrm{min} / 1.73 \mathrm{~m}^{2}\right)$ over the course of 2 years. Presently, 4 years following the development of irAIN, the patient remains off therapy and free of systemic disease with the exception of a single subcentimeter brain lesion that was treated with SRS. His most recent studies showed a $\mathrm{Cr}$ of $1.48 \mathrm{mg} / \mathrm{dL}\left(\mathrm{eGFR}\right.$ of $50 \mathrm{~mL} / \mathrm{min} / 1.73 \mathrm{~m}^{2}$ ), and a normal urine protein to $\mathrm{Cr}(\mathrm{P} / \mathrm{C})$ ratio of $<0.2$.

\section{Case 2}

The patient is a 70-year-old man with a history of stage II chronic kidney disease, type II diabetes (T2DM), and HTN, who initially presented with mRCC complicated by a level IV tumor thrombus, and synchronous metastases to the lungs and bone on March 2015. He was not a candidate for cytoreductive nephrectomy and a biopsy of his primary tumor revealed ccRCC. NG of the biopsy sample was 2, and no sarcomatoid or rhabdoid features were observed. He was initially treated with pazopanib and had a partial response lasting 8 months. At progression, he was started on an investigational HIF-2 $\alpha$ inhibitor PT2385, ${ }^{12}$ and this was followed by axitinib, but he was refractory to both therapies. At that point, he started nivolumab ( $3 \mathrm{mg} / \mathrm{kg}$ every 2 weeks). He received three doses of nivolumab with what was believed to be progression at the time, which was accompanied by clinical deterioration, resulting in transition to hospice care. Remarkably, the patient returned to clinic 7 months later with a significant reduction in tumor burden (figure 2, compare $\mathrm{C}$ and D; see also Ref. 13).

Several months after re-establishing care (approximately 9 months after starting nivolumab initially), nivolumab was reinitiated (240 mg every 2 weeks) due to mild progression. Three months later, his Cr increased from a baseline of 1.5 to 
1

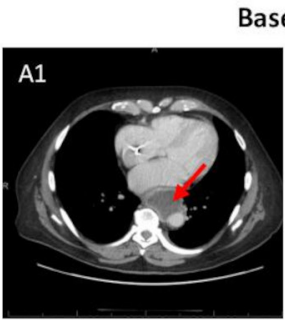

aseline
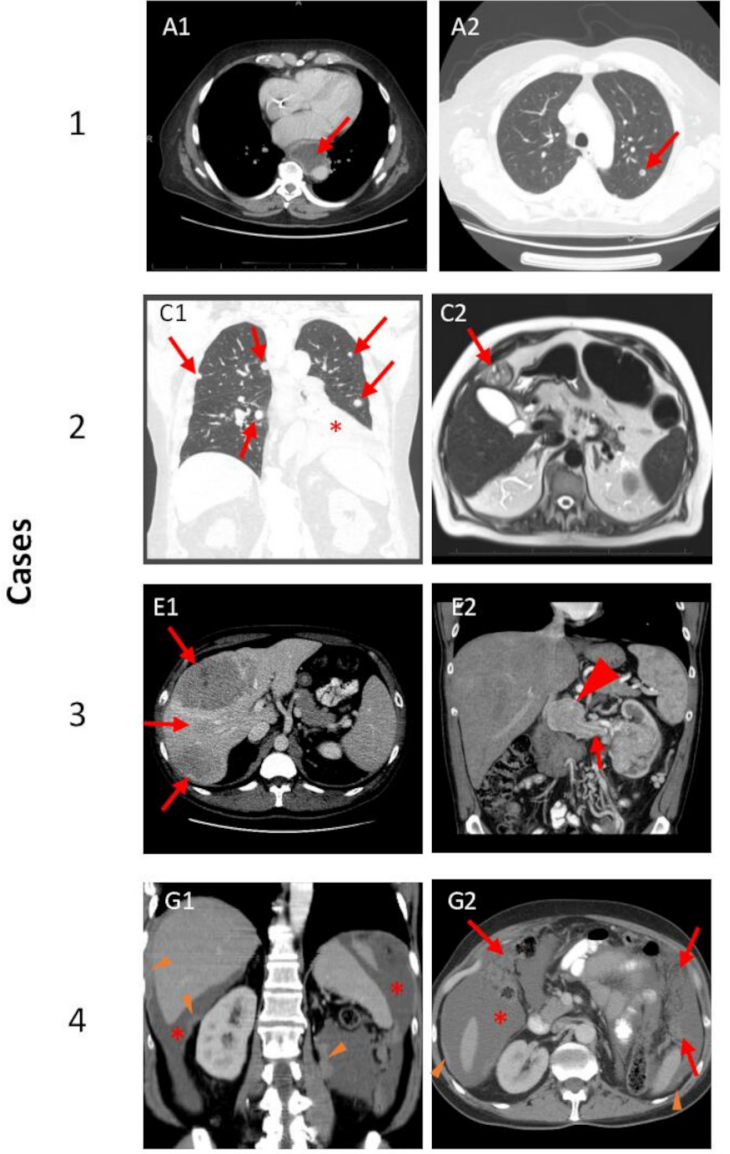

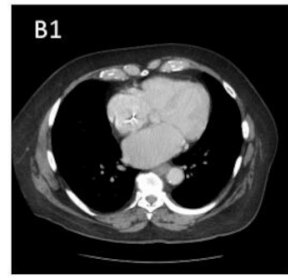

Post ICI
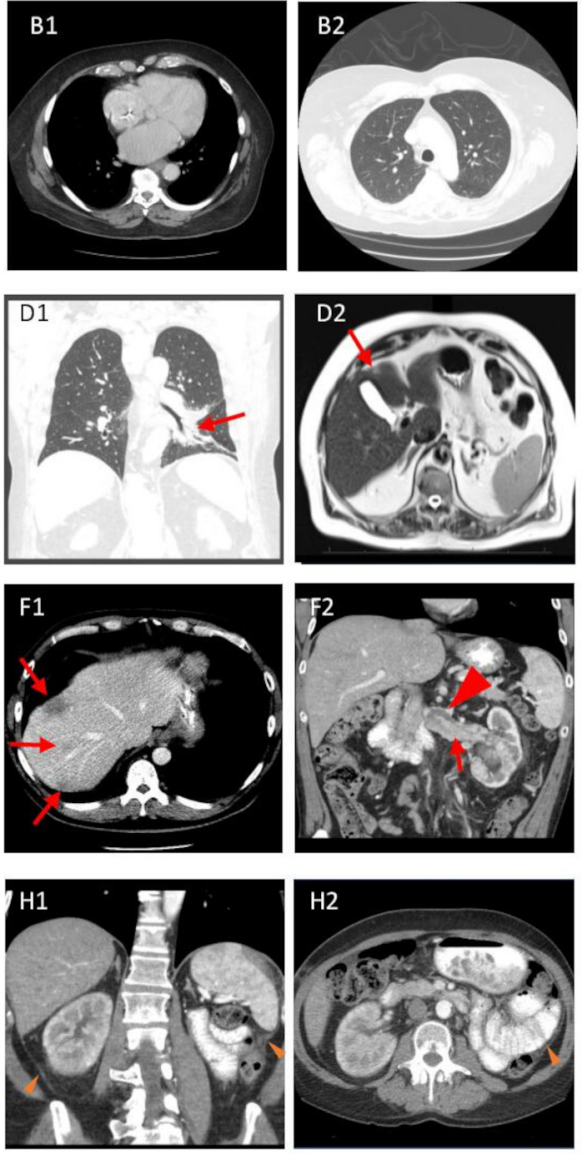

Figure $2 \mathrm{ICl}$-associated tumor changes. Case 1: Red arrows highlight representative lesions at baseline including a paraesophageal lymph node, which was biopsied (A1) and pulmonary nodule (A2). Post-ICl imaging (B1 and B2) demonstrate resolution of these lesions. Case 2: Red arrows show representative pulmonary $(C 1)$ and hepatic lesions (C2); red asterisk highlights left lower lobe consolidation/atelectasis secondary to metastatic lesion and pleural effusion (C1). Post-ICl imaging demonstrates substantial improvement of pulmonary disease (D1) and the liver lesion (D2). Case 3: Red arrows show large liver masses (E1) and tumor thrombus (E2) with mass-like enlargement of tumor thrombus at the confluence with the inferior vena cava (arrowhead). Post-ICl imaging shows significant shrinkage of liver metastases (F1) and tumor thrombus (F2). Case 4: Baseline imaging with peritoneal deposits (orange arrowheads), malignant ascites (red asterisk) (G1), and omental caking (red arrows) (G2). Post-ICl imaging demonstrates resolution of ascites and omental caking, as well as significant reduction in peritoneal deposits (orange arrowheads). $\mathrm{ICl}$, immune checkpoint inhibitor.

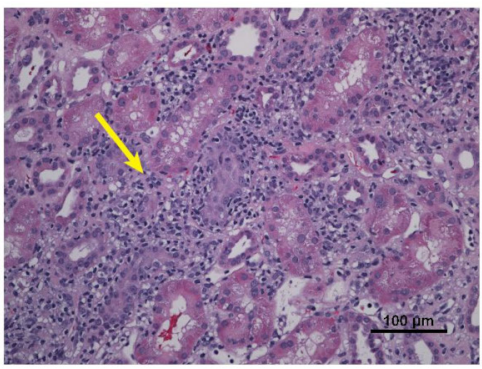

Case 1

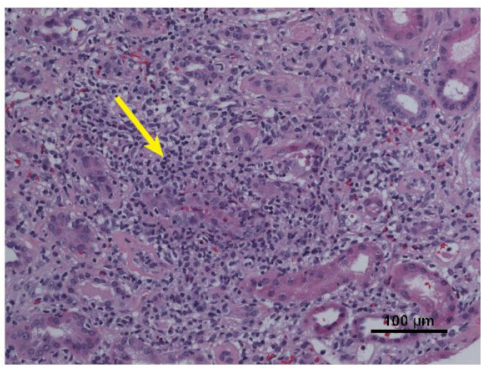

Case 2

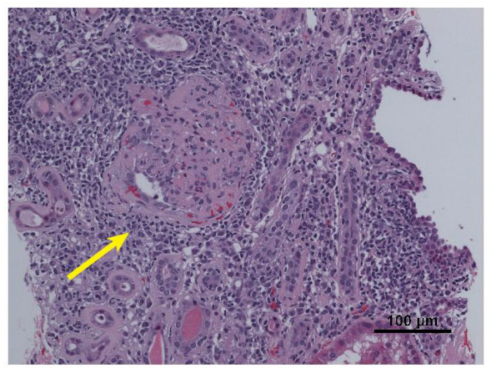

Case 4

Figure 3 Histological features of irAIN. H\&E stained sections from three biopsied cases showing a diffuse interstitial inflammatory infiltrate with prominent lymphocytes, a few plasma cells and occasional eosinophils. Focal lymphocytic tubulitis (yellow arrows) and focal tubular epithelial cell injury with mild interstitial edema was observed. Case 1 shows background focal segmental glomerulosclerosis likely unrelated to the acute presentation. Case 2 shows diffuse diabetic glomerulopathy with global and segmental glomerulosclerosis, consistent with the patient's history of T2DM and CKD. CKD, chronic kidney disease; $\mathrm{H} \& \mathrm{E}$, hematoxylin and eosin; irAIN, immune-related acute interstitial nephritis; T2DM, type II diabetes. 
$10 \mathrm{mg} / \mathrm{dL}$ (eGFR decreased from 42 to $5 \mathrm{~mL} / \mathrm{min} / 1.73 \mathrm{~m}^{2}$ ). This was accompanied by the development of proteinuria, with a urine $\mathrm{P} / \mathrm{C}$ ratio of 2.6 (previously $<0.2$ ). The patient was not oliguric or symptomatic. Ultrasound-guided biopsy of the kidney confirmed irAIN (figure 3). Nivolumab was stopped, methylprednisolone $500 \mathrm{mg}$ daily was administered intravenously for 3 days, and subsequently he received a prednisone taper starting at $120 \mathrm{mg}$ PO daily over 3 months. His renal function improved with a decrease in $\mathrm{Cr}$ to $1.85 \mathrm{mg}$ / $\mathrm{dL}\left(\mathrm{eGFR}, 36 \mathrm{~mL} / \mathrm{min} / 1.73 \mathrm{~m}^{2}\right.$ ) and urine $\mathrm{P} / \mathrm{C}$ of 2.3 with eventual decrease to 1.6. The patient was followed with active surveillance for an additional 3 months. Imaging at this time demonstrated progressive disease with new lung nodules and enlargement of liver lesions. He received a combination of lenvatinib and everolimus, on which he had stable disease lasting an additional year, and ultimately passed away due to complications of pneumonia.

\section{Case 3}

The patient is a 48-year-old otherwise healthy man who was initially diagnosed with RCC and a level II thrombus with bulky retroperitoneal lymphadenopathy and synchronous metastases to the liver. Biopsy of the primary tumor revealed ccRCC. NG on the biopsy was 3 and no sarcomatoid or rhabdoid features were appreciated. The patient was initially started on combination ipilimumab and nivolumab, with SBRT to the primary tumor through the RADVAX trial (NCT03065179). ${ }^{14}$ Following the second infusion of ipilimumab/nivolumab $(1 \mathrm{mg} / \mathrm{kg}$ and $3 \mathrm{mg} / \mathrm{kg}$, respectively), an elevation in Cr to $2.45 \mathrm{mg} / \mathrm{dL}$ from a baseline of $1.2 \mathrm{mg} / \mathrm{dL}$ (eGFR from $>60$ to $28 \mathrm{~mL}$ / $\min / 1.73 \mathrm{~m}^{2}$ ) was noted on routine labs. Additional ICI infusions were held, and the patient was started on $1 \mathrm{mg}$ / $\mathrm{kg}$ prednisone twice daily, with initial improvement in Cr. After 1 week, the prednisone dose was reduced to $1 \mathrm{mg} /$ $\mathrm{kg}$ once daily, but this resulted in a gradual rise in $\mathrm{Cr}$, prompting the addition of mycophenolate $1.5 \mathrm{~g}$ PO twice daily while the prednisone was gradually reduced over a period of 6 weeks. Four months later, immunosuppressants were stopped and kidney function normalized with a $\mathrm{Cr}$ of $1.28 \mathrm{mg} / \mathrm{dL}\left(\mathrm{eGFR}\right.$ of $\left.>60 \mathrm{~mL} / \mathrm{min} / 1.73 \mathrm{~m}^{2}\right)$. Imaging at that time showed a remarkable response, with shrinkage of all lesions (figure 2, compare E to F). The patient ultimately elected to undergo consolidative nephrectomy. Most recent surveillance imaging 2 years after initiating therapy demonstrates no evidence of disease and the patient continues to do well.

\section{Case 4}

This patient is a 58-year-old man with a medical history of HTN, T2DM, and coronary artery disease who initially presented with stage II papillary RCC (pRCC) at an outside institution and underwent a left radical nephrectomy. One year later, the patient developed ascites, was diagnosed with peritoneal carcinomatosis, and was referred to UTSW for management. Review of the pathology inhouse revealed pRCC, NG 3, without sarcomatoid or rhabdoid features. Cytological review of ascites fluid was consistent with metastatic
pRCC. The patient was started on combination ipilimumab $1 \mathrm{mg} / \mathrm{kg}$ and nivolumab $3 \mathrm{mg} / \mathrm{kg}$ every 3 weeks and a peritoneal drain was placed. Six weeks after starting therapy, ascites was markedly improved and the peritoneal drain was removed. After completing his fourth infusion, the patient developed AKI with a peak $\mathrm{Cr}$ of $2.65 \mathrm{mg} / \mathrm{dL}$, up from a baseline of $0.8 \mathrm{mg} / \mathrm{dL}$ (eGFR from $>60$ to $37 \mathrm{~mL} / \mathrm{min} / 1.73$ $\mathrm{m}^{2}$ ). Urinalysis at this time did not demonstrate proteinuria. Renal biopsy demonstrated inflammatory changes consistent with irAIN (figure 3), as well as IgA nephropathy with diffuse, global, granular mesangial positive staining by IgA (not shown). The patient was started on $1 \mathrm{mg} / \mathrm{kg}$ of prednisone which was tapered down over a period of 3 months. The patient's Cr gradually down trended to $1.74 \mathrm{mg} / \mathrm{dL}$ (eGFR, $49 \mathrm{~mL} / \mathrm{min} / 1.73 \mathrm{~m}^{2}$ ). Imaging 4 months following ICI demonstrated a remarkable improvement in the peritoneal carcinomatosis (figure 2, compare $\mathrm{G}$ and $\mathrm{H}$ ), and resolution of ascites.

\section{DISCUSSION}

Through a systematic database search, we identified three patients with mRCC who developed irAIN following ICI therapy for an incidence rate of $1.7 \%$. We also identified a fourth patient, diagnosed after the latest database update, through a query of oncologists at UTSW. ICI was held for all four patients and immunosuppressive therapy was started in three of four patients. All patients demonstrated at least a partial recovery of renal function, and none required renal replacement therapy. The presence of proteinuria was variable, with one patient (case 1) developing nephrotic range proteinuria and another with more modest impairment of renal function without detectable urine protein (case 4). Both the prevalence and clinical spectrum of irAIN in this series is consistent with studies of irAIN of pooled tumor types. $^{1516}$

Interestingly, the time to irAIN onset was quite variable. The two patients treated with nivolumab monotherapy developed irAIN as a late complication and only after reintroduction of nivolumab. Late onset irAEs may be attributable to antigenic spread following a successful antitumor response ${ }^{17}$ possibly resulting in the immune recognition of shared tumor and normal epitopes. Conversely, the two patients treated with combination ipilimumab/nivolumab developed irAIN within 3 months of starting therapy. This may be attributable to the increased rate of irAEs observed with dual ICI therapy relative to single agent therapy. ${ }^{26}$

IgA nephropathy was observed on the renal biopsy of Case 4. IgA nephropathy following PD-L1 inhibitor therapy has been reported, ${ }^{18}$ however, IgA nephropathy is also a recognized paraneoplastic syndrome of RCC; $;^{19}$ thus, causality is difficult to establish. Case 3 received concurrent SBRT to the primary renal lesion with combination ipilimum$a b+n i v o l u m a b ;$ however, it is unlikely that the kidney injury sustained was induced by radiation toxicity since renal toxicity following SBRT is uncommon and usually of late onset. ${ }^{820}$

All four patients demonstrated a deep response to therapy, with two patients demonstrating long term disease control 
off systemic therapy, one of which has had an ongoing response lasting 4years. Importantly, our cohort includes patients with pathologic and imaging findings associated with poor prognosis such as bone metastases (patient 2); large hepatic metastases (patient 3); and high grade pRCC with diffuse peritoneal carcinomatosis (patient 4). As a reference, responses rates with single agent nivolumab are $25 \%$, and $40 \%$ with combination therapy. ${ }^{21}$ Our data raises the possibility that irAIN may be correlated with improved outcomes in ICI-treated RCC, although the conclusions are obviously limited by the small sample size and retrospective nature of this report. While irAIN can develop following ICI therapy of different tumor types, we are unaware of any literature suggesting irAIN specifically correlates with improved oncologic outcomes in non-RCC tumors. ${ }^{71}{ }^{22-24}$ Conversely, we have identified two case reports in which patients with RCC demonstrated a prolonged response to ICI treatment following the development of irAIN. ${ }^{25} 26$ These data support the notion of antigenic overlap. We propose that oncological benefit following irAIN may be unique to RCC, and possibly due to shared epitopes between normal renal epithelium and RCC that drive a robust and sustained immune response. Finally, by identifying a second tumor type (besides melanoma) in which improved responses are associated with irAEs directed towards the tissue of origin, our data suggest that this phenomenon might be generalizable to other tumor types; that is, immune mediated adverse events of the tissue of origin might portend improved oncological outcomes, though further studies are needed to evaluate this notion.

\section{Author affiliations}

${ }^{1}$ Department of Internal Medicine, University of Texas Southwestern, Dallas, Texas, USA

${ }^{2}$ Kidney Cancer Program, Simmons Comprehensive Cancer Center, University of Texas Southwestern Medical Center, Dallas, Texas, USA

${ }^{3}$ Department of Pathology, University of Texas Southwestern Medical Center, Dallas, Texas, USA

${ }^{4}$ Lyda Hill Department of Bioinformatics, University of Texas Southwestern Medical Center, Dallas, Texas, USA

${ }^{5}$ Division of Nephrology, Department of Internal Medicine, University of Texas Southwestern Medical Center, Dallas, Texas, USA

${ }^{6}$ Department of Radiology, University of Texas Southwestern Medical Center, Dalla, Texas, USA

${ }^{7}$ Department of Radiation Oncology, University of Texas Southwestern Medical Center, Dallas, Texas, USA

Twitter Viral Patel @ViralMPatel19, Roy Elias @rmelias2, Venkat Malladi @ katatonikkat, Payal Kapur @P1kapu, Ivan Pedrosa @ivpedrosa, Hans Hammers @ HHammersMD and James Brugarolas @JBrugarolas and UTSW Kidney Cancer Program @KCPUTSW

Acknowledgements The authors acknowledge the patients and their families. This research was supported by NIH SPORE Grant P50CA196516 (JB, PK, RM, IP, $\mathrm{AC})$.

Contributors Conception and design: VP, RE, JB. Development of methodology: AC, VM, VP, RE, JB. Acquisition of data: VP, RE, JF, WS, AC, QC, VM, PK, RM, IP, HH, JB. Analysis and interpretation of data: VP, RE, JF, QC, PK, MV, IP, RH, JB. Writing, review, and/or revision of manuscript: VP, RE, WS, PK, MV, RM, IP, RH, HH, JB.

Funding This research was supported by the UTSW Kidney Cancer SPORE grant P50CA196516 (JB). The Kidney Cancer Explorer is supported by UTSW Kidney Cancer SPORE grant P50CA196516 and the Cancer Prevention and Research Institute of Texas award RP150596 (VM). RE receives support from an institutional award from the Burroughs Wellcome Fund. RH was funded by American Cancer
Society grant RSG-16-004-01-CCE. MV receives support from NIDDK grants UH3DK104655 and UH3DK114870.

Competing interests $\mathrm{JB}$ is an employee/paid consultant for Exelixis and Arrowhead; reports receiving commercial research grants from Arrowhead; and holds ownership interest (including patents) in Peloton Therapeutics/Merck, outside the submitted work. HH has served on an Advisory Board for the following companies: Bristol-Myers Squibb; Merck; Pfizer; Armo Biosciences; Novartis, Surface Oncology, Exelixis, Corvus and received a grant or contract from: BristolMyers Squibb and Merck, outside the submitted work. IP reports personal fees from Bayer Healthcare, personal fees from Health Tech International, other from Philips Healthcare, outside the submitted work.

\section{Patient consent for publication Obtained.}

Ethics approval Retrospective clinical data were collected in compliance with institutional guidelines after approval of the Institutional Review Board, protocol number STU 012 011-190.

Provenance and peer review Not commissioned; externally peer reviewed.

Open access This is an open access article distributed in accordance with the Creative Commons Attribution Non Commercial (CC BY-NC 4.0) license, which permits others to distribute, remix, adapt, build upon this work non-commercially, and license their derivative works on different terms, provided the original work is properly cited, appropriate credit is given, any changes made indicated, and the use is non-commercial. See http://creativecommons.org/licenses/by-nc/4.0/.

\section{ORCID iDs}

Roy Elias http://orcid.org/0000-0002-8555-4322

Joseph Formella http://orcid.org/0000-0002-1846-8133

Venkat Malladi http://orcid.org/0000-0002-0144-0564

Raquibul Hannan http://orcid.org/0000-0003-1282-056X

James Brugarolas http://orcid.org/0000-0002-8575-499X

\section{REFERENCES}

1 Howlader N, Noone A, Krapcho M, et al. Seer cancer statistics review, 1975-2016. Bethesda, MD: National Cancer Institute, 2019.

2 Motzer RJ, Tannir NM, McDermott DF, et al. Nivolumab plus ipilimumab versus sunitinib in advanced renal-cell carcinoma. $N$ Engl J Med 2018;378:1277-90.

3 Rini BI, Plimack ER, Stus V, et al. Pembrolizumab plus axitinib versus sunitinib for advanced renal-cell carcinoma. N Engl J Med 2019;380:1116-27.

4 Motzer RJ, Penkov K, Haanen J, et al. Avelumab plus axitinib versus sunitinib for advanced renal-cell carcinoma. $N$ Engl J Med 2019;380:1103-15.

5 Tannir NM, Frontera OA, Hammers HJ, et al. Thirty-month followup of the phase III CheckMate 214 trial of first-line nivolumab + ipilimumab $(\mathrm{N}+\mathrm{I})$ or sunitinib (S) in patients (PTS) with advanced renal cell carcinoma (aRCC). Journal of Clinical Oncology 2019;37:547.

6 Kennedy LB, Salama AKS. A review of cancer immunotherapy toxicity. CA Cancer J Clin 2020;70:86-104.

7 Das S, Johnson DB. Immune-Related adverse events and anti-tumor efficacy of immune checkpoint inhibitors. J Immunother Cancer 2019;7:306.

8 Berner F, Bomze D, Diem S, et al. Association of checkpoint inhibitorinduced toxic effects with shared cancer and tissue antigens in nonsmall cell lung cancer. JAMA Oncol 2019;5:1043-7.

9 Teulings H-E, Limpens J, Jansen SN, et al. Vitiligo-like depigmentation in patients with stage III-IV melanoma receiving immunotherapy and its association with survival: a systematic review and meta-analysis. J Clin Oncol 2015;33:773-81.

10 Indini A, Di Guardo L, Cimminiello C, et al. Immune-related adverse events correlate with improved survival in patients undergoing antiPD1 immunotherapy for metastatic melanoma. J Cancer Res Clin Oncol 2019;145:511-21.

11 Hua C, Boussemart L, Mateus C, et al. Association of vitiligo with tumor response in patients with metastatic melanoma treated with pembrolizumab. JAMA Dermatol 2016;152:45-51.

12 Courtney KD, Infante JR, Lam ET, et al. Phase I dose-escalation trial of PT2385, a first-in-class hypoxia-inducible factor- $2 \alpha$ antagonist in patients with previously treated advanced clear cell renal cell carcinoma. J Clin Oncol 2018;36:867-74.

13 Elias R, Kapur P, Pedrosa I, et al. Renal cell carcinoma pseudoprogression with clinical deterioration: to hospice and back. Clin Genitourin Cancer 2018;16:485-8. 
14 Hammers HJ, Vonmerveldt D, Ahn C, et al. Combination of dua immune checkpoint inhibition $(\mathrm{ICl})$ with stereotactic radiation (SBRT) in metastatic renal cell carcinoma (mRCC) (RADVAX RCC). J Clin Oncol 2020;38:614.

15 Cortazar FB, Kibbelaar ZA, Glezerman IG, et al. Clinical features and outcomes of immune checkpoint inhibitor-associated AKI: a multicenter study. J Am Soc Nephrol 2020;31:435-46.

16 Seethapathy $\mathrm{H}$, Zhao S, Chute DF, et al. The incidence, causes, and risk factors of acute kidney injury in patients receiving immune checkpoint inhibitors. Clin J Am Soc Nephrol 2019;14:1692-700.

17 Gulley JL, Madan RA, Pachynski R, et al. Role of antigen spread and distinctive characteristics of immunotherapy in cancer treatment. $J$ Natl Cancer Inst 2017;109:djw261.

18 Mamlouk O, Selamet U, Machado S, et al. Nephrotoxicity of immune checkpoint inhibitors beyond tubulointerstitial nephritis: single-center experience. J Immunother Cancer 2019;7:2.

19 Mimura I, Tojo A, Kinugasa S, et al. Renal cell carcinoma in association with IgA nephropathy in the elderly. Am J Med Sci 2009;338:431-2

20 Correa RJM, Louie AV, Zaorsky NG, et al. The emerging role of stereotactic ablative radiotherapy for primary renal cell carcinoma: a systematic review and meta-analysis. Eur Urol Focus 2019;5:958-69.
21 Motzer RJ, Escudier B, McDermott DF, et al. Nivolumab versus everolimus in advanced renal-cell carcinoma. $N$ Engl J Med 2015;373:1803-13.

22 Fujii T, Colen RR, Bilen MA, et al. Incidence of immune-related adverse events and its association with treatment outcomes: the MD Anderson cancer center experience. Invest New Drugs 2018;36:638-46.

23 Judd J, Zibelman M, Handorf E, Julia J, Matthew Z, Elizabeth H, et al. Immune-Related adverse events as a biomarker in nonmelanoma patients treated with programmed cell death 1 inhibitors. Oncologist 2017;22:1232-7.

24 Cortellini A, Chiari R, Ricciuti B, et al. Correlations between the immune-related adverse events spectrum and efficacy of anti-PD1 immunotherapy in NSCLC patients. Clin Lung Cancer 2019;20:237-47.

25 Kus T, Aktas G. Durable response after interruption of nivolumab in patients with metastatic renal cell carcinoma: is renal toxicity a marker to predict the benefit of nivolumab? J Oncol Pharm Pract 2020;26:1000-4.

26 Xipell M, Victoria I, Hoffmann V, et al. Acute tubulointerstitial nephritis associated with atezolizumab, an anti-programmed death-ligand 1 (PD-L1) antibody therapy. Oncoimmunology 2018;7:e1445952. 